\title{
Agonist activation of estrogen receptor beta (ER $\beta)$ sensitizes malignant pleural mesothelioma cells to cisplatin cytotoxicity
}

\author{
Giulia Pinton ${ }^{1}$, Arcangela G Manente ${ }^{1}$, Antonio Daga ${ }^{2}$, Michele Cilli ${ }^{2}$, Maurizio Rinaldi ${ }^{1}$, Stefan Nilsson ${ }^{3,4}$ \\ and Laura Moro ${ }^{1 *}$
}

\begin{abstract}
Background: Estrogen receptor (ER) $\beta$ acts as a tumor suppressor in malignant mesotheliomas.

Methods: Here we explored the anti-proliferative and anti-tumorigenic efficacy of the selective ER $\beta$ agonist, KB9520, in human mesothelioma cell lines in vitro and in a mesothelioma mouse model in vivo.

Results: KB9520 showed significant anti-proliferative effect in ERß positive human malignant pleural mesothelioma cells in vitro. Selective activation of ERß with KB9520 sensitized the cells to treatment with cisplatin, resulting in enhanced growth inhibition and increased apoptosis. Furthermore, in CD1 nude mice mesothelioma tumor growth was significantly inhibited when KB9520 was added on top of the standard of care chemo combination cisplatin/ pemetrexed, as compared to the cisplatin/pemetrexed alone group. Importantly, KB9520 exerted a protective effect to cisplatin toxicity in the non-malignant mesothelium derived MET5A cells.
\end{abstract}

Conclusions: Together, the data presented suggest that selective targeting of ERß may be an efficacious stand-alone treatment option and/or become an important add-on to existing malignant mesothelioma therapy.

Keywords: Estrogen Receptor $\beta$, Malignant mesothelioma, Receptor agonist, Cisplatin, Therapy

\section{Background}

Malignant pleural mesothelioma (MPM) is an aggressive cancer associated with exposure to asbestos. Currently rates of MPM are rising and estimates indicate that the incidence of MPM will peak within the next 10-15 years in the western world, while in Japan the peak is predicted not to occur until 40 years from now [1,2]. Although the use of asbestos has been banned in many countries around the world, production of and the potentially hazardous exposure to asbestos is still present with locally high incidences of mesothelioma [3]. Today a new manmade material, carbon nanotubes (CNTs), has arisen as a concern; CNTs may display 'asbestos-like' pathogenicity with mesothelioma induction potential $[4,5]$. The pharmacology of CNTs, including effects on immune responses

\footnotetext{
* Correspondence: moro@pharm.unipmn.it

'Department of Pharmaceutical Sciences, University of Piemonte Orientale

"A. Avogadro", Lgo Donegani 2, 28100 Novara, Italy

Full list of author information is available at the end of the article
}

and tissue accumulation, is therefore intensely investigated; "it is better to be safe than sorry" [6].

MPM is an extremely difficult disease to treat, with a median overall survival ranging between 9 and 17 months, regardless of disease stage $[7,8]$. The combination of pemetrexed and cisplatin has been established as the current standard of care (SOC) but only $40 \%$ of treated patients show response to this therapy, with an overall median survival of 12.1 months [9].

There is an urgent need for new targeted therapy for clinical management of MPM that can stop progression and stabilize the disease or ideally erase the tumor, either as add-on to SOC, to improve treatment efficacy and reduce chemoresistance and toxicity, or as monotherapy for patients whose performance status does not allow aggressive treatment.

Rapid advances in the understanding of cancer biology are leading to identification of new targets for cancer treatment and personalized therapy is rapidly becoming 
a reality with the aim of improving survival and quality of life for cancer patients [10-12].

The expression of estrogen receptor beta (ER $\beta)$ in malignant pleural and peritoneal mesothelioma correlates with longer patient survival and is an independent prognostic factor $[13,14]$. Our group recently demonstrated that $70-80 \%$ of MPMs express ER $\beta$, which acts as a tumor suppressor, inhibiting MPM cell proliferation and invasiveness $[13,15]$.

$E R \beta$ is the second ER subtype identified in several human tissues traditionally believed to be ER negative [16]. ER $\beta$ is expressed and demonstrated to exert anti-proliferative effects in different preclinical in vitro and in vivo models of human cancers, for example, breast, colon, prostate, lymphoma, and pleural mesothelioma [13,15,17-25]. Moreover, ER $\beta$ is proposed to mediate the beneficial clinical effects in ER $\alpha$ negative breast cancer patients [26], in malignant intraperitoneal mesothelioma [27], and in the prevention of colon cancer in women on menopausal hormone therapy (MHT) [28].

Drugs that selectively target ER $\beta$ might be safer than non-selective estrogens, which are associated with increased risk of breast, endometrial and ovarian cancer in women and the development of prostate cancer in men. These serious side effects of non-selective estrogens are mediated by the ER $\alpha$ subtype [29-32]. Several synthetic and natural ER $\beta$-selective compounds have been identified [19,33-36] that have shown promising anti-tumorigenic efficacy in preclinical cancer models [37-43]. Therefore, drugs with selectivity for ER $\beta$ might prove promising in the the development of novel, targeted therapies for the clinical management of human cancers.

In the present study, we characterized the efficacy of KB9520, a selective ER $\beta$ agonist, to inhibit MPM cell growth in vitro and in vivo. Moreover, we investigated the possibility of an additive or synergistic effect between KB9520 and the SOC regimen (cisplatin/pemetrexed) for treatment of MPM.

\section{Results \\ KB9520-mediated ER $\beta$ activation affects MPM cell proliferation}

The growth inhibitory effect of different concentrations of KB9520 (range 1-100 nM) was tested on ER $\beta$ positive REN mesothelioma cells (Figure 1A). KB9520 significantly $(p \leq 0,05)$ reduced cell growth and viability in a concentration-dependent manner, with highest efficacy at $10 \mathrm{nM}$. For comparison we also examined the concentration-dependent growth inhibitory effect of $17 \beta$-estradiol (E2) on REN cells (Figure 1B). The antiproliferative activity of KB9520 as single agent was then assessed in the non-malignant mesothelium derived MET5A cells and in the malignant mesothelioma REN, MMB, H2596, and MSTO-211H cell lines, respectively
(Figure 1B). KB9520 significantly $(\mathrm{p} \leq 0,05)$ inhibited proliferation of the REN and MMB cells, whereas no inhibitory effect was observed in the MET5A cells, despite high endogenous levels of ER $\beta$. The H2596 cells express very low levels of ER $\beta$ which explains their weaker response to KB9520. As expected, the ER $\beta$ negative MSTO- $211 \mathrm{H}$ cells and the ER $\beta$ silenced REN and MMB cells showed no response to KB9520 treatment. However, transient transfection of the MSTO- $211 \mathrm{H}$ cells with an ER $\beta$ expression vector sensitized also these cells to KB9520 (Figure 1B). In addition, increasing the expression of ER $\beta$ in $\mathrm{H} 2596$ by transient transfection with an ER $\beta$ expression vector increased the response to KB9520 also in these cells (data not shown).

\section{KB9520 significantly increases the effect of cisplatin/ pemetrexed in vitro and in vivo}

To test if KB9520 influences MPM cell response to chemotherapy in vitro, we explored the effect of adding $10 \mathrm{nM}$ KB9520 to the cisplatin/pemetrexed chemo combination (at their respective IC50 concentrations) on REN cell viability. As shown in Figure 2A, the triple combination KB9520/cisplatin/pemetrexed (10 nM/100 $\mu \mathrm{M} /$ $22 \mu \mathrm{M}$, respectively) was superior $(\mathrm{p} \leq 0.05)$ to either KB9520 or cisplatin/pemetrexed treatment alone.

To confirm the role of agonist activated ER $\beta$ in the enhanced response to chemotherapy, the effect of KB9520 on top of the cisplatin/pemetrexed combination was evaluated also in the mesothelioma in vivo mouse model. Six weeks old CD1 nude male mice were inoculated intra peritoneum with $2 \times 10^{6}$ REN cells (4 groups, 10 animals per group). Prior to inoculation, the MPM cells were transduced with a lentiviral vector carrying the luciferase gene, to allow imaging in live mice. Treatment of the animals was initiated fifteen days after cell inoculation when tumor take-rate in the peritoneal cavity was $100 \%$ in all animal groups. The ER $\beta$-selective agonist KB9520 was administrated on day 15 through 35 by subcutaneous injection at $10 \mathrm{mg} / \mathrm{kg} /$ day. Untreated animals were subcutaneously dosed with empty vehicle. Two groups were treated at day 18 and 25 with $5 \mathrm{mg} / \mathrm{kg}$ cisplatin followed by 5 days treatment with $150 \mathrm{mg} / \mathrm{kg}$ pemetrexed (days 19-23 and 26-30), alone or in combination with KB9520 (see the treatment schedule in Table 1). KB9520 alone treated mice produced a similar decrease in tumor dimensions as the cisplatin/pemetrexed treated group compared to vehicle controls at day 10 (data not shown). After 21 days of treatment, we observed a statistically significant reduction in tumor growth in the group treated with KB9520 plus cisplatin/ pemetrexed as compared to the vehicle, KB9520, and cisplatin/pemetrexed groups, respectively (Figure 2B, C). Furthermore, these statistical significant differences were confirmed when the tumor growth curves were compared. 

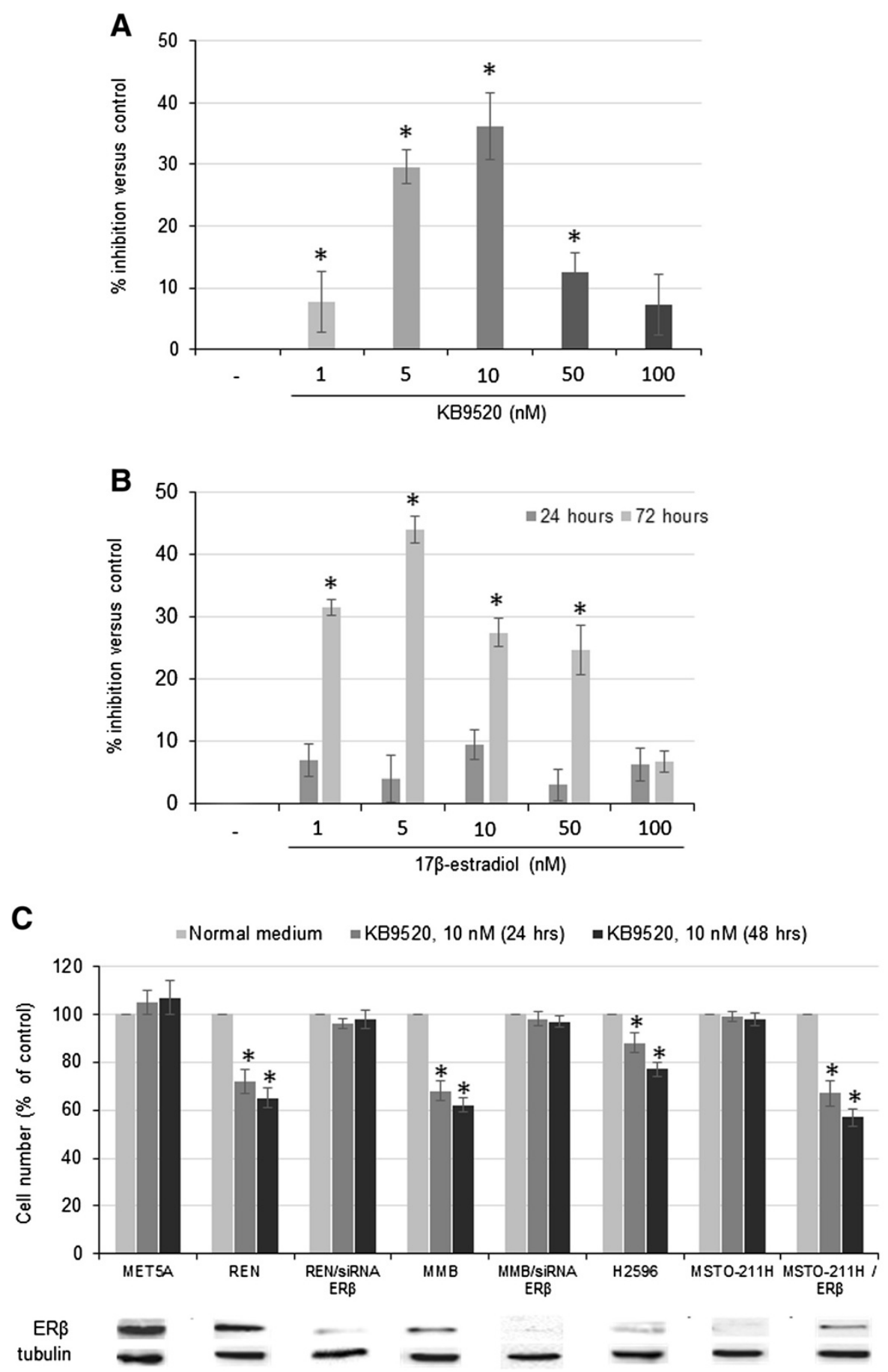

Figure 1 KB9520 mediated ER $\beta$ activation affects MPM cells proliferation. A) Bar graph shows the percentage of growth inhibition of REN cells after 24 hours treatment with different concentrations of KB9520 (range 1-100 nM) versus untreated cells. B) Bar graph shows the percentage of growth inhibition of REN cells after 24 and 72 hours treatment with different concentrations of 17ß-estradiol (range 1-100 nM) versus untreated cells. C) Bar graph shows the percentage of growth inhibitory effect of $10 \mathrm{nM} \mathrm{KB9520}$ at 24 and 48 hours in mesothelium derived cells (MET5A), mesothelioma cells with different levels of endogenous ER $\beta$ expression (REN, MMB, H2596 and MSTO-211H), ER $\beta$ silenced REN and MMB cells (REN/siRNA ERß, MMB/siRNA ERß) and MSTO-211H/ERß cells, which were transiently transfected to express human ER $\beta$. Western blots, below the bar graph, show ER $\beta$ protein expression for each cell line. Tubulin was included as a loading control. Each graph is representative of three independent experiments. Each bar represents mean \pm s.d. ${ }^{*} p \leq 0.05$. 

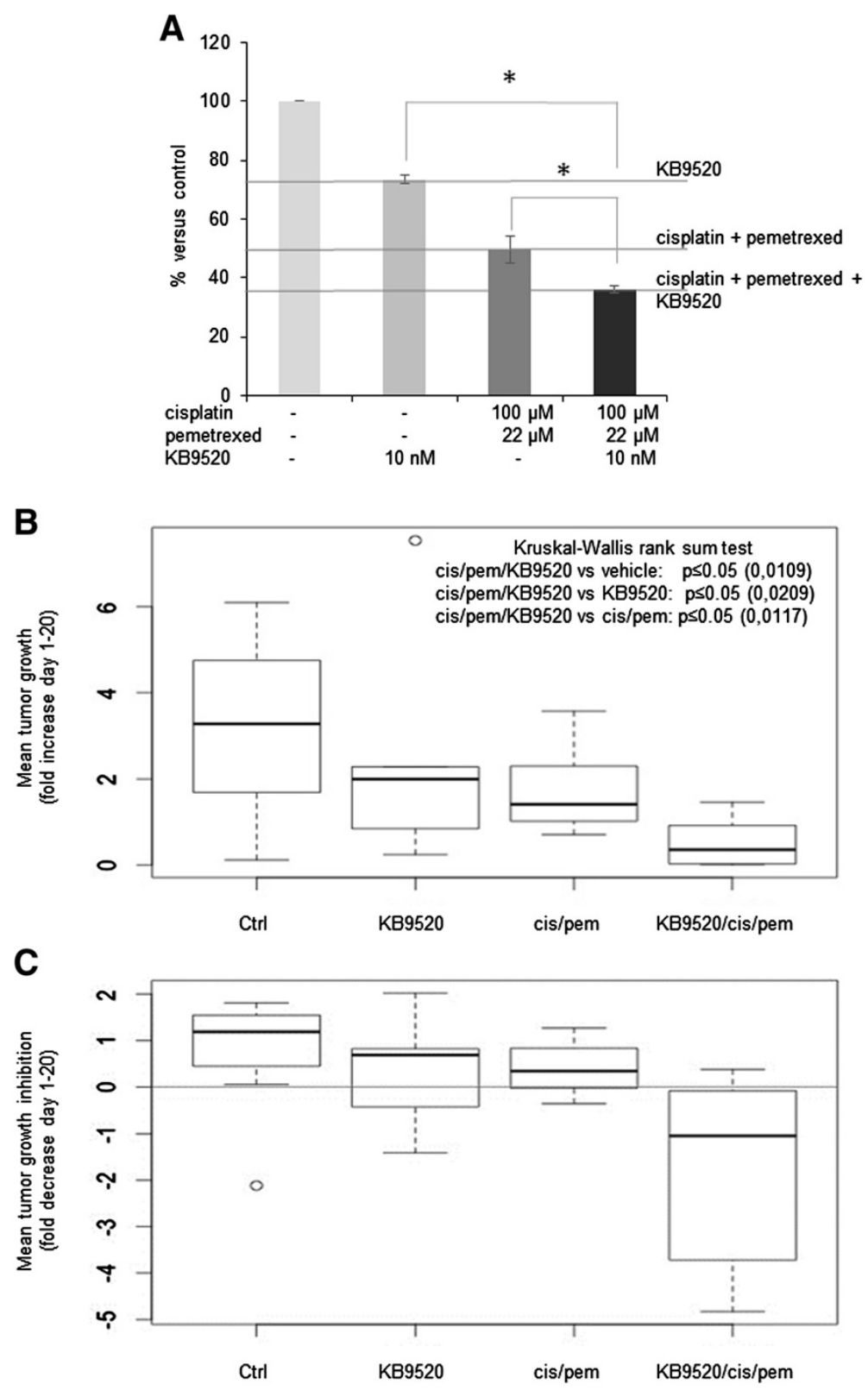

Figure 2 KB9520 significantly increases cisplatin/pemetrexed effects in vitro and in vivo. A) Percentage of viable REN cells exposed for 24 hours to $10 \mathrm{nM} \mathrm{KB9520}$ alone or in combination with cisplatin $(100 \mu \mathrm{M})$ and pemetrexed $(22 \mu \mathrm{M})$, versus untreated cells. Graph is representative of three independent experiments. Each bar represents mean \pm s.d. ${ }^{*} p \leq 0.05$. B) and $\mathbf{C}$ ) Box plots of the 4 different treatment groups (10 mice/group) showing in vivo mean tumor growth (B) and mean tumor growth inhibition (C) evaluated after 21 days of treatment. The thick segments represent the medians while the upper and lower borders of each rectangle represent the quartiles. Bars show minimum and maximum values for each group, outliers are identified by a small circle. Kruskal-Wallis rank sum test (in B) confirms that combination treatment significantly reduced tumor growth, compared with control or single treatments.

Table 1 Treatment schedule of the in vivo experiment

\begin{tabular}{|c|c|c|c|c|c|c|c|c|c|c|c|c|c|c|c|c|c|c|c|c|c|c|}
\hline Day 1 & Treatment & Day 15 & 16 & 17 & 18 & 19 & 20 & 21 & 22 & 23 & 24 & 25 & 26 & 27 & 28 & 29 & 30 & 31 & 32 & 33 & 34 & Day 35 \\
\hline & KB9520 (10 mg/kg) & $x$ & $x$ & $x$ & $x$ & $x$ & $x$ & $x$ & $x$ & $x$ & $x$ & $x$ & $x$ & $x$ & $x$ & $x$ & $x$ & $x$ & $x$ & $x$ & $x$ & $x$ \\
\hline \multirow[t]{2}{*}{ REN cells injected } & cisplatin (5 mg/kg) & & & & $x$ & & & & & & & $x$ & & & & & & & & & & \\
\hline & pemetrexed (150 mg/kg) & & & & & $x$ & $x$ & $x$ & $x$ & $x$ & & & $x$ & $x$ & $x$ & $x$ & $x$ & & & & & \\
\hline
\end{tabular}


Treatment with KB9520 was not toxic as assessed by monitoring changes of mice body weights during drug administration (data not shown). 35 days after MPM cell inoculation all animals were sacrificed and tumors were dissected and immediately frozen.

\section{Biological sustainability of KB9520}

Since the in vivo plasma half-life of KB9520 is only approximately 1 hour in mice (data not shown) we decided to investigate the biological sustainability and mechanism of action of KB9520 in vitro to better understand its anti-tumorigenic activity and synergism with cisplatin/ pemetrexed in vivo. Firstly, we tested the anti-proliferative response to brief exposures (1, 2, 4, 8, 16 and 24 hours) to $10 \mathrm{nM}$ KB9520 on the ER $\beta$ positive REN cells (Figure 3A). An exposure of 2 hours presented significantly $(\mathrm{p} \leq 0.05)$ increased inhibitory activity relative to 1 hour exposure and exposures longer than 8 hours. Successively, the 2 hours exposure to KB9520 was characterized further.
Cells were treated with different concentrations of KB9520 (0.4, 2 and $10 \mathrm{nM})$ for 2 hours followed by wash-off and continued growth in normal medium (without KB9520) for an additional 24, 48 and 72 hours (Figure 3B). Control cultures were maintained in normal medium only. The duration of inhibitory effect on REN cell proliferation sustained for at least 24 hours irrespective of concentration of KB9520 used in the 2-hours pre-treatment period. The largest anti-proliferative effect was, however, observed with the highest KB9520 concentration used. After 24 hours the cells slowly regained proliferative activity and from $\sim 48$ hours post KB9520 pre-treatment, their proliferative rates were similar to that of REN cells cultivated in normal medium from start of study.

\section{KB9520 pre-treatment sensitizes REN cells to cisplatin}

To explore the sequence of drug administration and its effect on cell proliferation we performed add-on or
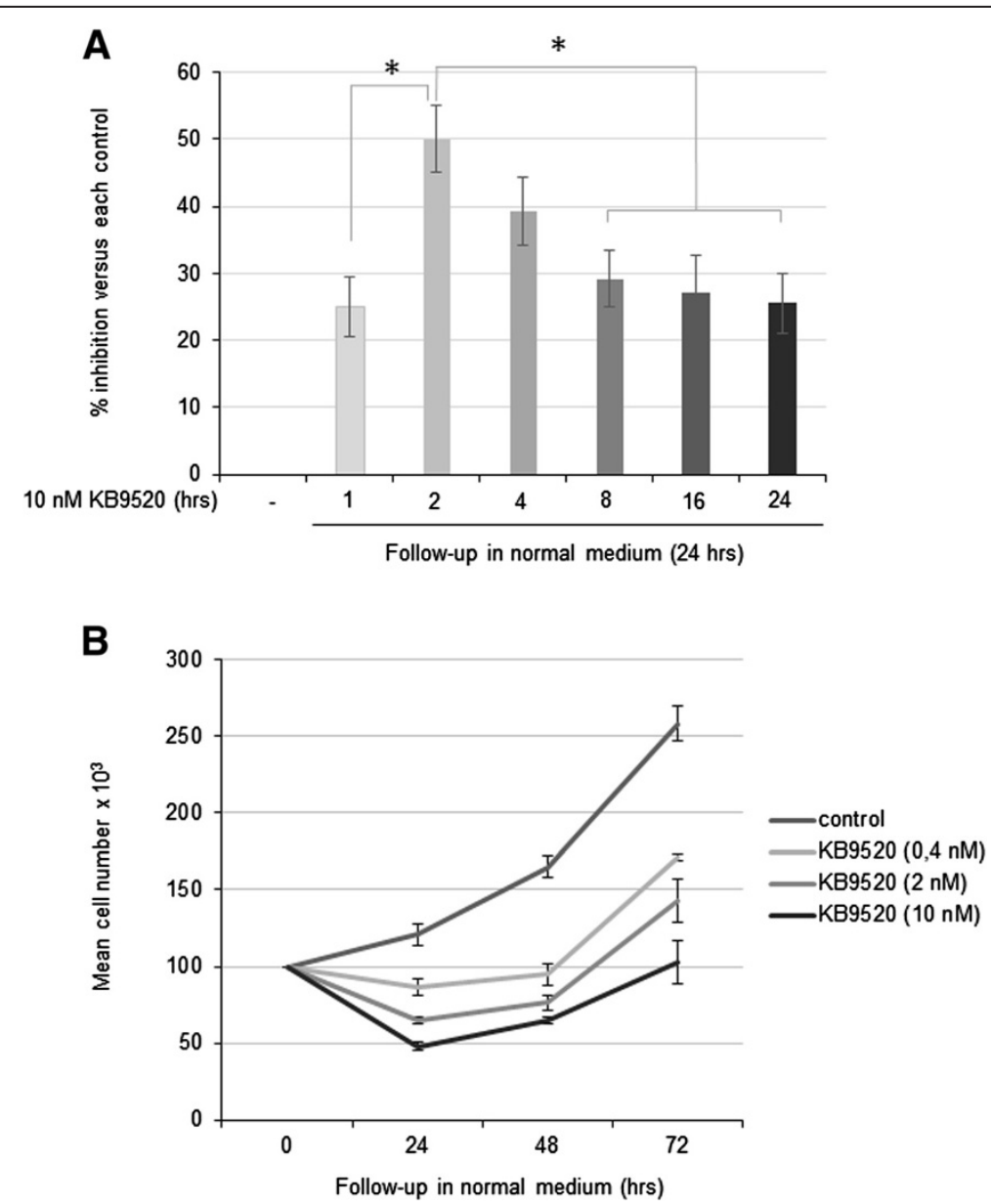

Figure 3 Biological sustainability of KB9520. A) Percentage of growth inhibition in REN cells after 1, 2, 4, 8, 16 or 24 hours pre-treatment with $10 \mathrm{nM} \mathrm{KB9520}$ followed by wash-off and continued growth in normal medium for an additional 24 hours. B) Mean number of REN cells pre-exposed for 2 hours to normal medium or 0.4, 2, 10 nM KB9520 followed by wash-off and continued growth for additional 24-, 48- and 72 hours in normal medium, respectively. Each graph is representative of three independent experiments. Each point represents mean \pm s.d. ${ }^{*} \mathrm{p} \leq 0.05$. 
wash-off experiments (Figures 4 and 5). In the first study $100 \mu \mathrm{M}$ cisplatin was added to REN cell cultures pretreated for 2, 4, 8 and 12 hours with $10 \mathrm{nM}$ KB9520 (Figure 4A). The enhanced anti-proliferative effect of cisplatin was time-dependent with the greatest inhibitory effect obtained adding cisplatin within 2 hours of KB9520 pre-treatment.

The reverse order of pre-treatment starting with cisplatin for 2, 4, 8 and 12 hours, respectively, prior to add-

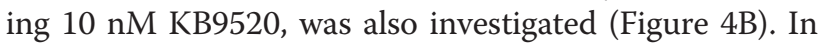
contrast to pre-treatment with KB9520 before adding cisplatin, pre-treatment with cisplatin prior to KB9520 addition did not result in synergistic inhibition of cell growth and viability.
Next we explored the combination of 2 hours KB9520 pre-treatment with different concentrations of cisplatin (range $20-100 \mu \mathrm{M}$ ) (Figure 5A). The most efficacious anti-proliferative effect was observed when KB9520 pretreatment was combined with the highest concentration of cisplatin $(100 \mu \mathrm{M})$. Interestingly, 2 hours pre-treatment with KB9520 in combination with $20 \mu \mathrm{M}$ cisplatin was as efficacious as $100 \mu \mathrm{M}$ cisplatin alone.

Also the effect of combining KB9520 with pemetrexed was explored in vitro (Figure 5B). Results of 2 hours

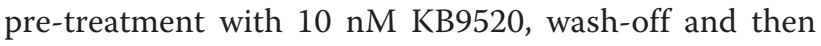
continued growth in normal medium, or medium supplemented with pemetrexed at different concentrations (range 5-22 $\mu \mathrm{M}$ ) for an additional 24 hours are shown.
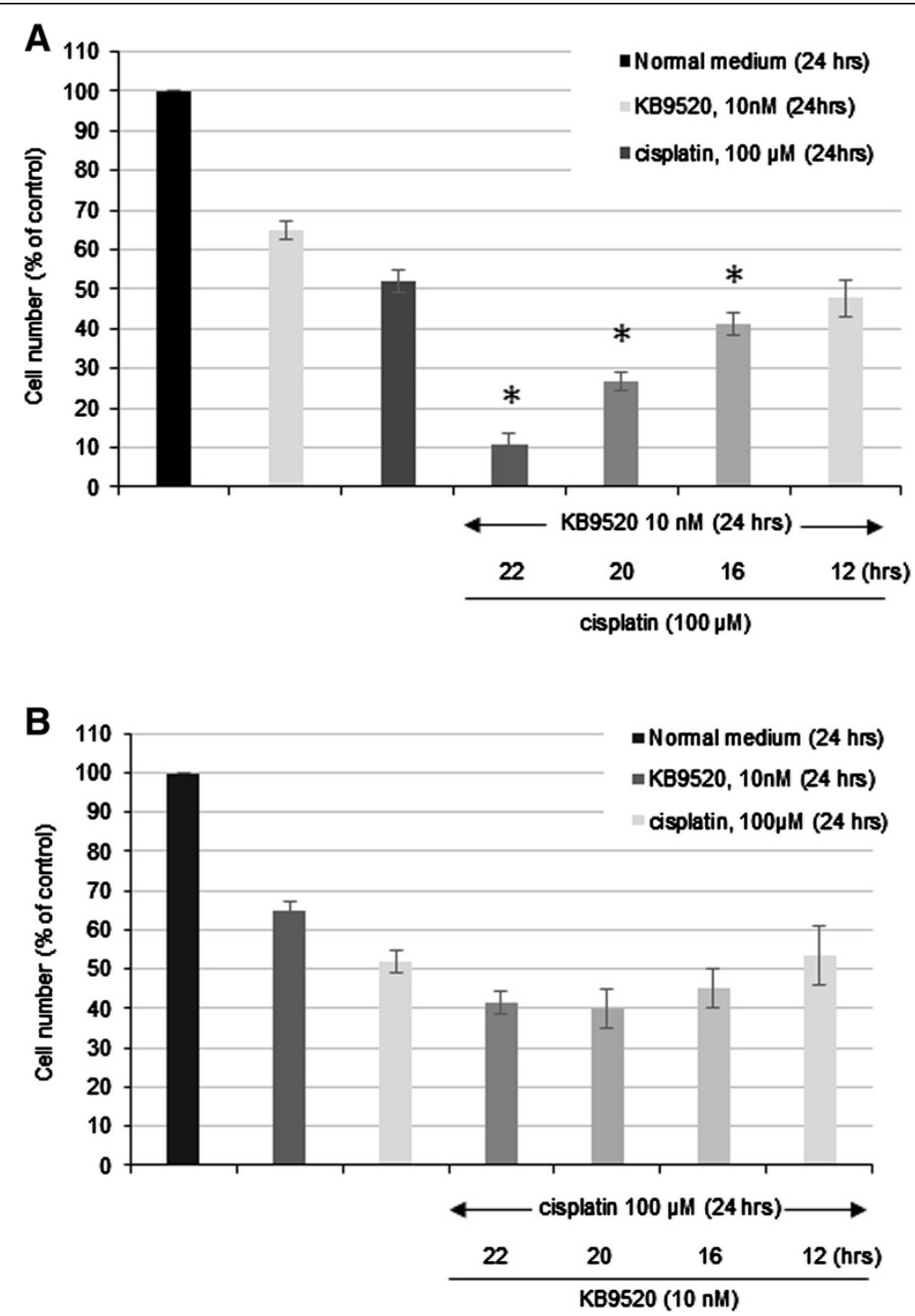

Figure 4 KB9520 pre-treatment synergizes with cisplatin. A) Effect of adding of cisplatin (100 $\mu$ M) 2, 4, 8 or 12 hours after start of KB9520 $(10 \mathrm{nM})$ treatment on REN cell viability. B) Effect of adding KB9520 (10 nM) 2, 4, 8 or 12 hours after start of cisplatin treatment (100 $\mu \mathrm{M})$ on REN cell viability. Each graph is representative of three independent experiments. Each point represents mean \pm s.d. * $\mathrm{p} \leq 0.05$. 

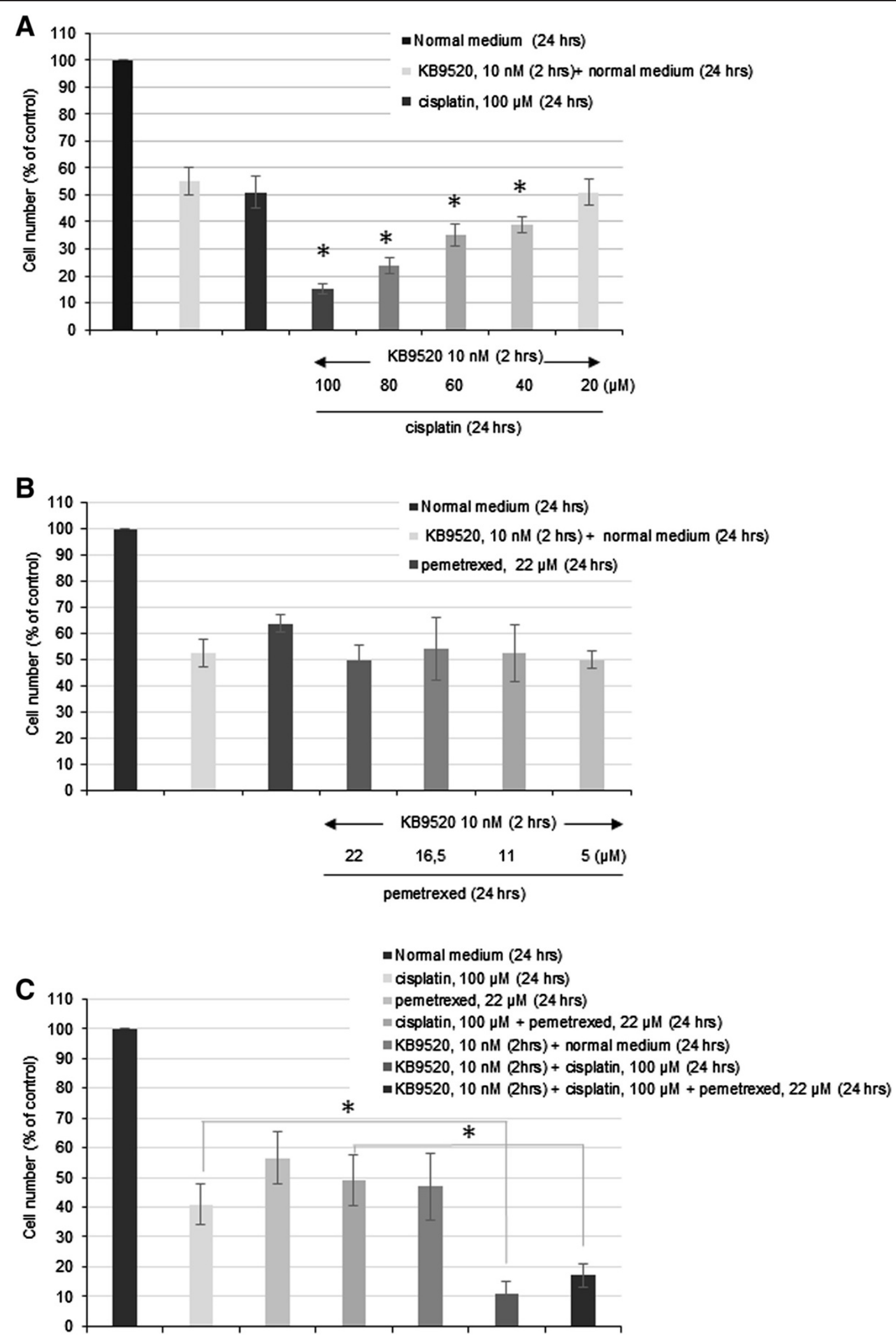

Figure $\mathbf{5}$ (See legend on next page.) 
(See figure on previous page.)

Figure 5 KB9520 sensitizes REN cells to cisplatin and the standard of care chemo combination. Effect on REN cell viability after 2 hours pre-treatment with KB9520 (10nM) followed by wash-off and continued growth in normal medium supplemented with different concentrations of A) cisplatin $(20-100 \mu \mathrm{M})$, for additional 24 hours, B) pemetrexed (5-22 $\mu \mathrm{M})$, for additional 24 hours and C) cisplatin (100 $\mu \mathrm{M})$ or the cisplatin $(100 \mu \mathrm{M}) /$ pemetrexed $(22 \mu \mathrm{M})$ combination, for an additional 24 hours. Each graph is representative of three independent experiments. Each point represents mean \pm s.d. ${ }^{*} p \leq 0.05$.

No synergism or additive effect was observed with KB9520 in combination with pemetrexed compared to KB9520 or pemetrexed alone. Finally, the effect of pretreating REN cells with $10 \mathrm{nM}$ KB9520 for 2 hours prior to adding the cisplatin/pemetrexed chemo combination was explored in vitro (Figure $5 \mathrm{C}$ ). As shown, adding cisplatin or the cisplatin/pemetrexed combination to REN cells pre-treated for 2 hours with KB9520 resulted in a strong synergistic inhibitory effect on REN cell growth and viability.

In summary, our data suggest that KB9520 acts as a chemosensitizer through ER $\beta$, increasing the anti-tumorigenic efficacy of cisplatin or the cisplatin/pemetrexed combination on malignant mesothelioma.

Mechanism of KB9520 sensitization to cisplatin cytotoxicity REN cells were treated for 24 hours with $100 \mu \mathrm{M}$ cisplatin or pre-treated 2 hours with 10 nM KB9520 followed by wash-off and continued growth in normal medium \pm $100 \mu \mathrm{M}$ cisplatin, for additional 24 hours. After treatments, cells were stained with propidium iodide and analyzed for cellular DNA content by flow cytometry. Pretreatment with KB9520 for 2 hours followed by 24 hours cisplatin treatment resulted in significant and efficient block in the G0/G1 phase and inhibition of cells entering the S-phase of the cell cycle compared to any other treatment (data reported in Table 2 represent mean $\pm s$. d. $(n=3)$ of the percentage of cells in each phase of the cell cycle). Moreover, a significant higher percentage of dead cells were found in wells pre-treated with KB9520 followed by cisplatin compared to other treatment regimens. A plausible explanation for the higher number of dead cells in the KB9520/cisplatin treated cells was induction of apoptosis. As expected from the cell cycle analysis and percentage of dead cells, 2 hours KB9520 treatment, prior to addition of cisplatin, had the greatest effect on the appearance of cleaved PARP1 (Figure 6A). Interestingly, neither cisplatin nor KB9520 alone resulted in significant PARP1 cleavage. As increased AKT activity has been implicated in the control of proliferation, apoptosis and cisplatin resistance [44], we analyzed its activation status following different treatments. As shown in Figure 6A, KB9520 treatment significantly reduced AKT phosphorylation both in the absence and in the presence of cisplatin. The mechanism for the combined effect of KB9520 and cisplatin on AKT pathway modulation, PARP1 cleavage and increased cell death needs further studies.

\section{KB9520 pre-treatment protects MET5A cells to cisplatin cytotoxicity}

Cisplatin is widely used in the treatment of various human solid tumors, but it is also associated with significant toxicity. We therefore tested the effect of KB9520 on cisplatin toxicity in the normal mesothelium derived cell line MET5A (Figure 6B, C). MET5A cells showed higher sensitivity to cisplatin treatment than REN cells, with an IC50 of $25 \mu \mathrm{M}$ (Figure 6B). As previously described for REN cells, we treated MET5A cells with 10 nM KB9520 for 2 hours followed by wash-off and incubation in normal medium alone or in the presence of three concentrations of cisplatin $(25,50,100 \mu \mathrm{M})$ for additional 24 hours. As reported in Figure 6B, KB9520 alone had no effect on MET5A cell proliferation or viability, while a protective effect was observed when KB9520 pre-treatment was combined with low concentrations of cisplatin. As previously described for REN cells, we analyzed PARP1 cleavage as an indicator of cell death. Western-blot analysis (Figure 6C) revealed that KB9520 pre-treatment reduced the percentage of cleaved PARP1 in cells exposed to all concentrations of cisplatin, in accordance with data obtained on cell viability. Regarding phosphorylated AKT, KB9520 had no effect on basal pAKT levels in the MET5A control cells but it antagonized the cisplatin-mediated inhibition of AKT activation (Figure 6C). AKT pathway activation is associated with anti-apoptotic effects and cell survival and together with the effects of KB9520 on reduced PARP1 cleavage,

Table 2 Cell cycle analysis of REN cells

\begin{tabular}{lcccc}
\hline Treatment/cell cycle phase & Go/G1 & S & G2/M & Dead cells (sub G1) \\
\hline Control & $39 \pm 1,9$ & $33 \pm 2,4$ & $25 \pm 0,5$ & $3 \pm 1$ \\
100 HM cisplatin (24 hrs) & $39 \pm 0,9$ & $30 \pm 2,3$ & $23 \pm 3,2$ & $8 \pm 2$ \\
10 nM KB9520 (2 hrs) + normal medium (24 hrs) & $36 \pm 1,9$ & $29 \pm 0,9$ & $27 \pm 0,9$ & $8 \pm 2$ \\
10 nM KB9520 (2 hrs) $+100 \mu M$ cisplatin (24 hrs) & $48 \pm 0,8$ & $2 \pm 2$ & $28 \pm 2,9$ & $22 \pm 1$ \\
\hline
\end{tabular}




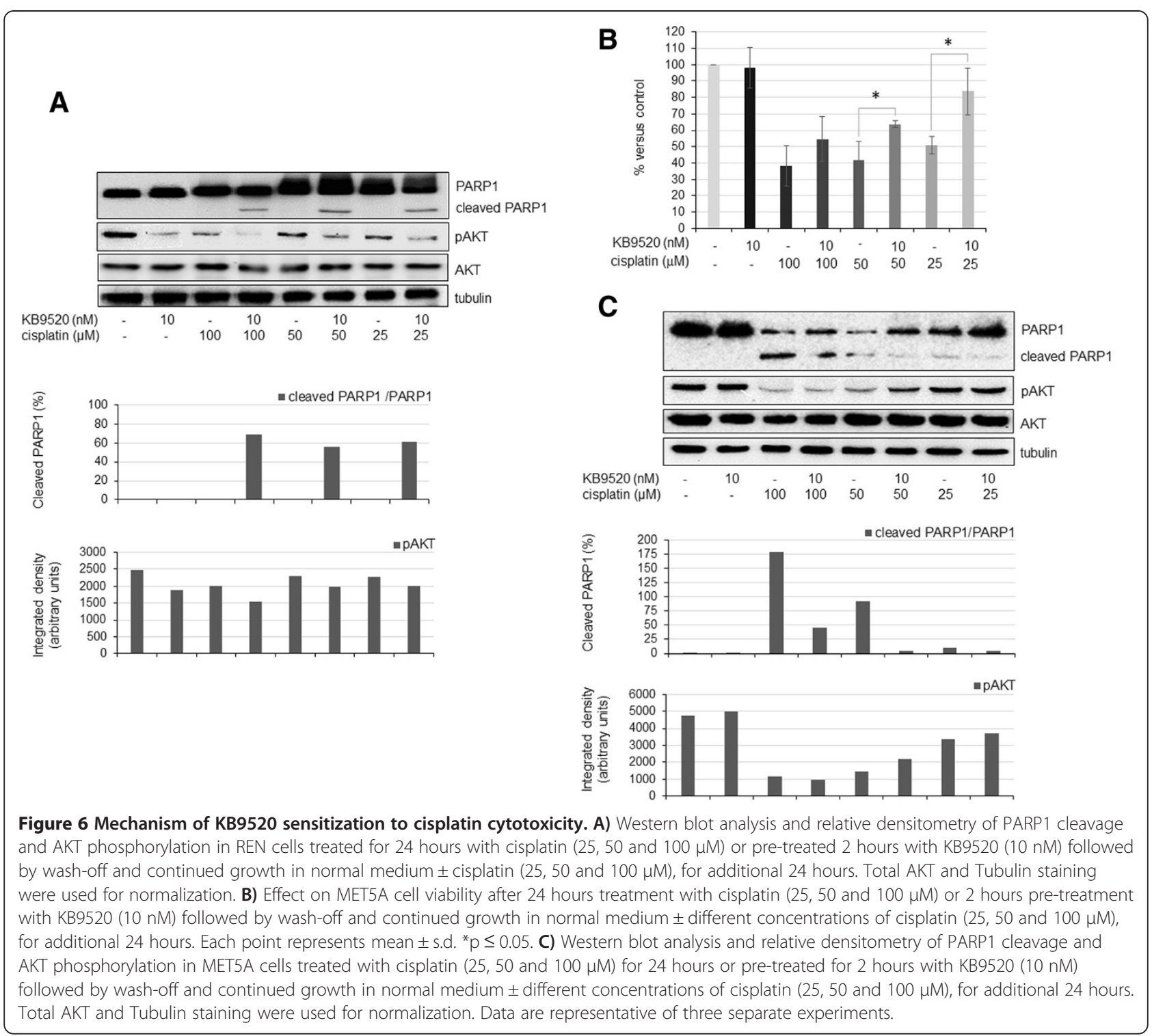

this may explain the KB9520-mediated decrease in cisplatin cytotoxicity in the non-malignant MET5A cells.

\section{Discussion}

MPM is typically refractory to current treatment options using chemotherapy. In a first line setting, pemetrexed in combination with cisplatin has been accepted as an almost universal standard. In the second line setting, various chemotherapy agents have been used, either as monotherapy or as part of polytherapy, but none has been successfully validated.

A recently published review, based on results of a meta-analysis of unresectable MPM, suggests that response rate and survival are greater for combination therapy than for single-agent regimens, and that platinum-containing regimens have greater efficacy than non-platinum-containing combinations, confirming that platinum-based chemotherapy remains the most effective treatment for patients with MPM [45].

Our group has recently demonstrated that ER $\beta$ exerts a key role as a tumor-suppressor gene in MPM [13,15] and its activation may also explain the gender difference clinically observed for the prognosis of MPM.

In this study we report that activating ER $\beta$ with the highly selective ligand KB9520 resulted in a concentration and time-dependent inhibition of REN malignant mesothelioma cell growth in vitro. Both KB9520 and E2 displayed bell-shaped responses with growth inhibition at low doses and opposite effect at high doses. Bell-shaped responses to hormones are not unusual phenomena; "hormesis" is a biphasic dose response phenomenon characterized by a low dose stressful stimulation and a high dose adaptive response (inhibition) that increases the resistance of the cell to evoked stress [46,47]. Both ER 
subtypes, $\alpha$ and $\beta$, exert genomic and no-genomic effects which may explain the observed biphasic responses of KB9520 and E2. Other plausible explanation could be the cyclic on/off process of ER on promoters [48,49] leading to degradation of the ER $\beta$ protein.

Although KB9520 and E2 had similar growth inhibitory effect on the REN cells there was a difference in time to maximal effect, 24 hours for KB9520 and 72 hours for E2. This time to effect difference between KB9520 and E2 may depend on the different ER $\beta$ conformational change/ s induced by KB9520 and E2, respectively, which in turn may have an effect on co-regulatory protein interactions including type of co-regulatory complex, stoichiometry and kinetics.

Evaluation of the anti-proliferative effect of KB9520 on a collection of human malignant mesothelioma cell lines showed a strict dependence on ER $\beta$ expression and that the growth inhibitory efficacy was related to the level of ER $\beta$ expressed. Lack of anti-proliferative effect of KB9520 in the ER $\beta$ positive non-malignant mesothelium derived MET5A cells is difficult to understand and needs further investigation. However, the difference in the regulatory mechanisms/signaling pathways for cell growth, survival and metabolism between non-malignant and malignant cells may somehow influence the activity of ER $\beta$ and its susceptibility to respond to an ER $\beta$ agonist. Differences in post-translational modification/s of ER $\beta$ and/or cell compartment localization are plausible explanations for activity or no activity of ER $\beta$ in malignant compared to non-malignant cells. Of note though, the non-malignant MET5A cells did respond to KB9520 when stressed by cisplatin.

The enhanced growth inhibitory effect of cisplatin/ pemetrexed in combination with KB9520 in REN cells in vitro translated to synergistic anti-tumorigenic activity in vivo. Treatment with KB9520 in combination with cisplatin/pemetrexed in vivo had greater efficacy than either treatment alone and caused a significantly reduced tumor load compared to vehicle treated animals at the end of the treatment period. Moreover, the triple combination shrunk the tumor volume even below the tumor volume at the start of treatment.

Also in a second human malignant mesothelioma cell line, MMP [13], KB9520 in complex with cisplatin resulted in synergistic growth inhibition compared to KB9520 or cisplatin alone in vitro (data not shown). Moreover, similar to the effect in REN cells, the combination of KB9520 and cisplatin in the MMP cells decreased the level of phosphorylated AKT and increased the levels of cleaved PARP1 (data not shown).

That a brief exposure of malignant mesothelioma cells to KB9520 (2 hours) elicited a stronger growth inhibitory effect in vitro compared to continuous exposure is suggestive of a hit-and-run mechanism. The long-lived biological activity of KB9520 ( $\geq 24$ hours) together with a hit-andrun type of mechanism adds to our understanding of the in vivo tumor inhibitory efficacy of KB9520 despite its short plasma half-life of approximately 1 hour in mice.

The PI3K/AKT signaling pathway is aberrantly active and plays a critical role in cell cycle progression and cell survival in human MPM including the sensitivity to cisplatin [50]. We have previously demonstrated a role of AKT activation in MPM cell response to cisplatin [44]. Here we show that KB9520 significantly reduced pAKT levels both in vitro and in vivo (data not shown), which, at least in part, may explain the observed sensitization to cisplatin cytotoxicity. The observed reduction in AKT phosphorylation warrant further investigation; it could be due to either modulation of the rate of both protein kinase and phosphatase activities or to AKT post-translational modification that could affect its localization and/or activation status.

The order of drug administration may sometimes be very important for optimal therapeutic efficacy [51,52]. Exposure of REN cells to KB9520 prior to cisplatin resulted in synergistic inhibition of malignant mesothelioma cell proliferation and survival whereas the reverse order of drug exposure did not even result in additive efficacy. Furthermore, KB9520 preconditioned malignant mesothelioma cells to low-concentration cisplatin cytotoxicity; combination of KB9520 with $20 \mu \mathrm{M}$ cisplatin was as efficacious as $100 \mu \mathrm{M}$ cisplatin alone. Thus, these data imply that KB9520, through ER $\beta$, acts as a chemosensitizer increasing cisplatin cytotoxicity in human malignant mesothelioma cells.

Chemotherapy and in particular cisplatin is widely used in the treatment of various human solid tumors. However, cisplatin is associated with serious toxicity, which limits its use; the majority of patients diagnosed with MPM are older than 65 years and their health condition may therefore not allow the standard chemo dosing regimen of cisplatin/pemetrexed. To investigate the cytotoxic effect of the cisplatin/KB9520 combination in non-malignant mesothelial cells we treated the mesothelium-derived MET5A cells with various concentrations of cisplatin in the presence or absence of KB9520. In contrast to the effect in the malignant REN cells KB9520 diminished the toxicity of cisplatin in the MET5A cells, in part explained by the reduced PARP1 cleavage and increased pAKT levels. It has been described that ER can increase PI3K/AKT activity by interaction with the p85 subunit of PI3K [53,54]. However, if that explains the increased pAKT levels in the MET5A cells needs to be explored in more detail.

\section{Conclusions}

In summary, in this report we have shown that MPM cell proliferation and tumor growth can be effectively suppressed by selective agonist activation of ER $\beta$. We 
have also shown that KB9520 acts as a chemosensitizer through activation of ER $\beta$ and that the order of drug administration in combination with cisplatin/pemetrexed is essential for the synergistic efficacy observed in vitro and in vivo. KB9520 had no cytotoxic effect in the ER $\beta$ expressing non-malignant mesothelium derived MET5A cells. In contrast, it diminished cisplatin cytotoxicity in these cells. Thus, combination of KB9520 with SOC (cisplatin/pemetrexed combination) may increase the sensitivity of MPM tumors to the SOC regimen in patients and perhaps result in higher response rates, extended progression free survival (PFS) and prolonged overall survival (OS), without adding toxicity. Furthermore, combination with KB9520 may allow milder SOC (cisplatin) regimen without loss of anti-tumor efficacy and thereby may become an option for patients that cannot tolerate the standard and more aggressive cisplatin/pemetrexed dose regimen.

\section{Methods}

\section{Reagents and antibodies}

The monoclonal antibodies specific for $\alpha$-Tubulin, PARP1 and the polyclonal antibody specific for ER $\beta$ were purchased from Santa Cruz Biotechnology (Santa Cruz, CA, USA). Phospho-AKT (pSer473) was from Cell Signaling Technology (Beverly, MA, USA), anti-mouse and anti-rabbit IgG peroxidase conjugated antibodies and chemical reagents were from Sigma-Aldrich (St Louis, MO, USA). ECL was from Amersham Pharmacia Biotech (Uppsala, Sweden). Nitrocellulose membranes and protein assay kit were from Bio-Rad (Hercules, CA, USA). Culture media, sera, antibiotics and LipofectAMINE transfection reagent were from Invitrogen (Carlsbad, CA, USA). The ER $\beta$ selective agonist KB9520 (see Additional file 1: Figure S1) was designed and synthesized by Karo Bio (Huddinge, Sweden). (KB9520 has been described previously [36,41-43]. The compound can be obtained following contact with Karo Bio AB [stefan.nilsson@karobio.se] and after signing of a Material Transfer Agreement together with a detailed protocol of planned study. A fee covering the cost of compound synthesis will be charged).

\section{Cell cultures and transfection}

The epithelioid MPM derived REN cell line, used as the principal experimental model in this investigation, was isolated, characterized and kindly provided by Dr. Albelda S.M. (University of Pennsylvania, Philadelphia; PA, USA). Cells were characterized by BMR Genomics s.r.l. using the PowerPlex 18D System kit. The biphasic MSTO-211H and the mesothelial MET5A cell lines were obtained from the Istituto Scientifico Tumori (IST) Cell-bank, Genoa, Italy; the MMB cell line derived from pleural effusions of patients with MPM and stabilized in culture [55]; the H2596 cell line produced by Dr. H. I. Pass from surgical specimens derived from patients with resected MPM [56] were kindly provided by Dr. W. Thomas (RCSI, Dublin, IRL) in 2011. Cells were grown in RPMI medium supplemented with $10 \% \mathrm{FBS}, 100 \mu \mathrm{g} / \mathrm{ml}$ streptomycin and $10 \mu \mathrm{g} / \mathrm{ml}$ penicillin at $37^{\circ} \mathrm{C}$ in a humidified environment containing 5\% CO2. Mycoplasma infection was excluded by the use of Mycoplasma PlusTM PCR Primer Set kit from Stratagene (La Jolla, CA, USA). Cells grown to $80 \%$ confluence in tissue culture dishes were transiently transfected with the pCNX2 plasmid expressing human wild type ER $\beta$ (Addgene, Cambridge, MA, USA) using LipofectAMINE reagent as described by the manufacturer.

Gene silencing was achieved using an ER $\beta$-specific shRNA lentiviral plasmid (pLKO.1-puro) by Sigma (St Louis, MO, USA) or specific siRNAs by Qiagen (Hilden, Germany).

\section{Proliferation assays}

Cells were seeded at a density of $10 \times 10^{4}$ cells/well in 6well plates in RPMI medium supplemented with $10 \%$ FBS, $100 \mu \mathrm{g} / \mathrm{ml}$ streptomycin and $10 \mu \mathrm{g} / \mathrm{ml}$ penicillin and incubated over-night at $37^{\circ} \mathrm{C}$ in a humidified environment containing 5\% CO2 to allow adherence. Following treatment cells were trypsinized and stained with Trypan blue. The number of cells considered viable (unstained cells) was counted in a Bürker haemocytometer within 5 minutes after staining.

\section{Wash-off experiments}

Cell cultures were pretreated with KB9520 for 1-16 hours (depending on experiment) followed by wash-off and then replenished with normal growth medium \pm cisplatin, pemetrexed or cisplatin/pemetrexed. Total incubation time was 24-72 hours (depending on experiment). Control cultures were maintained in normal growth medium without added drug. The number of viable cells was determined as described. For details of each experiment see figure legends.

\section{Add-on experiments}

In the add-on experiments the second drug was added directly to the cell culture medium without wash-off of the first drug. Total incubation time was 24 hours. Control cultures were maintained in normal growth medium without added drug. The number of viable cells was determined as described.

\section{Cell lysis and immunoblot}

Cells were extracted with $1 \%$ NP-40 lysis buffer (1\% NP40, $150 \mathrm{mM} \mathrm{NaCl}, 50 \mathrm{mM}$ Tris- $\mathrm{HCl}$ pH $8.5 \mathrm{mM}$ EDTA, $\left.10 \mathrm{mM} \mathrm{NaF}, 10 \mathrm{mM} \mathrm{Na} \mathrm{P}_{2} \mathrm{O}_{7}, 0.4 \mathrm{mM} \mathrm{Na}_{3} \mathrm{VO}_{4}\right)$ with freshly added protease inhibitors $(10 \mu \mathrm{g} / \mathrm{ml}$ leupeptin, $4 \mu \mathrm{g} / \mathrm{ml}$ pepstatin and $0.1 \mathrm{Unit} / \mathrm{ml}$ aprotinin). Lysates were centrifuged at $13.000 \times \mathrm{g}$ for 10 minutes at $4^{\circ} \mathrm{C}$ and 
the supernatants were collected and assayed for protein concentration with the Bio-Rad protein assay method.

Proteins were separated by SDS-PAGE under reducing conditions. Following SDS-PAGE, proteins were transferred to nitrocellulose, reacted with specific antibodies and then detected with peroxidase-conjugate secondary antibodies and chemiluminescent ECL reagent. Densitometric analysis was performed using the GS 250 Molecular Image (Bio-Rad).

\section{Cell cycle analysis}

For cell cycle/apoptosis analysis, $5 \times 10^{5}$ cells were seeded in tissue culture plates and treated with $10 \mathrm{nM}$ KB9520, $100 \mu \mathrm{M}$ cisplatin or the combination of the two drugs for 24 hours at $37^{\circ} \mathrm{C}$ in a $5 \% \mathrm{CO} 2$ atmosphere. After incubation, detached and suspended cells were harvested in complete RPMI and centrifuged at $500 \times \mathrm{g}$ for 10 minutes. Pellets were washed with PBS, fixed in ice-cold $75 \%$ ethanol at $4^{\circ} \mathrm{C}$, treated with $100 \mathrm{mg} / \mathrm{mL}$ RNAse A for 1 hour at $37^{\circ} \mathrm{C}$, stained with $25 \mu \mathrm{g} / \mathrm{mL}$ propidium iodide and finally analyzed by using a flow cytometer FACS (Becton Dickinson, San Jose, CA, USA) and Modfit software (Verity Software House, Topsham, ME, USA).

\section{In vivo experiments}

Animals

CD1 nude mice (males, 6 weeks old; Charles River, Calco, Italy) received intra-peritoneal (i.p.) injections of $2 \times 10^{6}$ luciferase transduced REN cells in $0.5 \mathrm{~mL}$ of RPMI medium. After anesthetization and i.p. injections of $0.3 \mathrm{~mL}$ of $15 \mathrm{mg} / \mathrm{mL}$ D-luciferin, tumor dimension and localization of luminescent cells was monitored using the In Vivo Imaging System (IVIS ${ }^{\circledR}$ ) system 100 series (Xenogen Corporation, Hopkinton, MA, USA). Regions of interest were identified around the tumor sites and were quantified as total photon counts using Living Image software (Xenogen Corporation). The values of tumor sizes were obtained by subtracting luminescence signals of each weekly measurement by the average of all animals within a treatment group on the $15^{\text {th }}$ day after inoculation (day when treatment started). To evaluate treatment toxicity, mice were weighed at the start and end of treatments. Mice were killed and necropsied after 20 days of treatment. In vivo experiments were approved by Istituto Scientifico Tumori (Genoa, Italy) ethical committee and conform to the relevant regulatory standards. Mice were maintained and handled under aseptic conditions, and were allowed access to food and water ad libitum.

\section{Drug administration}

An elapse of 15 days was allowed for the formation of detectable tumor nodules, assessed by IVIS $^{\oplus}$ imaging. Mice were then weighed and stratified into treatment groups of ten animals. Treatment protocols were done from the $15^{\text {th }}$ day to the $35^{\text {th }}$ day, and mice were analyzed weekly by IVIS ${ }^{\odot}$ imaging to assess tumor growth. One dose of KB9520 was used (10 mg/kg/day). KB9520 was dissolved in the vehicle (5\% DMSO/40\% PEG 400/ $55 \%$ water) and administrated once daily (days 15-35) by sub-cutaneous administration. $5 \mathrm{mg} / \mathrm{kg}$ cisplatin solution (Ebewe Italia srl, Roma, Italy) was administrated subcutaneously at day 18 and 25, respectively, and $150 \mathrm{mg} / \mathrm{kg}$ pemetrexed (dissolved in isotonic saline) (Eli Lilly, Houten, Nederland) was injected subcutaneously at days 19-23 and 26-30, respectively. Untreated animals were dosed with empty vehicle. At day 35 mice from the four groups were euthanized and necropsied. Tumors growing in the peritoneum were excised, and one part of the tumor tissues was immediately frozen and stored at $-80^{\circ} \mathrm{C}$ for subsequent analysis.

\section{Statistical analysis}

Statistical evaluation of the differential analysis was performed by one way ANOVA and Student's t-test. The threshold for statistical significance was set at $\mathrm{p} \leq 0.05$. The statistical analysis of in vivo experiments was done by using $\mathrm{R}$ [57]. To compare different groups we used the non-parametric Kruskal-Wallis test; if differences were found significant ( $\mathrm{p} \leq 0.05$ ) we subsequently applied the Wilkoxon rank sum test to do the pair wise comparisons. To compare tumor growth curves we used the StatMod package.

\section{Additional file}

Additional file 1: Figure S1. Concentration-dependent induction of reporter gene expression in 293 cells genetically engineered to express the human estrogen receptor alpha (hERa) and human estrogen receptor beta 1 (hERß) [58].

\section{Competing interests}

$\mathrm{SN}$ is an employee at Karo Bio AB. The remaining authors declare that they have no competing interests.

\section{Authors' contributions}

GP and AGM carried out cellular and molecular studies and made substantial contributions to analysis and interpretation of data, AD and MC performed in vivo studies. MR conducted statistical analysis. SN and LM participated in the design and coordination of the study and drafted the manuscript. All authors have read and approved the final manuscript.

\section{Acknowledgements}

Work was funded by Karo Bio Research Foundation (Huddinge, Sweden) (LM, grant 2012).

\section{Author details}

'Department of Pharmaceutical Sciences, University of Piemonte Orientale "A. Avogadro", Lgo Donegani 2, 28100 Novara, Italy. ${ }^{2}$ IRCCS San Martino-IST, 16132 Genova, Italy. ${ }^{3}$ Karo Bio AB, Novum, S-141 57 Huddinge, Sweden. ${ }^{4}$ Department of Biosciences and Nutrition, Karolinska Institutet, Novum, S-141 57 Huddinge, Sweden. 
Received: 15 April 2014 Accepted: 23 September 2014

Published: 2 October 2014

\section{References}

1. Robinson BM: Malignant pleural mesothelioma: an epidemiological perspective. Ann Cardiothorac Surg 2012, 1(4):491-496.

2. Prazakova S, Thomas PS, Sandrini A, Yates DH: Asbestos and the lung in the 21st century: an update. Clin Respir J 2013, 8(1):1-10.

3. Stayner L, Welch LS, Lemen R: The worldwide pandemic of asbests-related diseases. Annu Rev Public Health 2013, 34:205-216.

4. Donaldson K, Poland CA, Murphy FA, Macfarlane M, Chernova T, Schinwald A: Pulmonary toxicity of carbon nanotubes and asbestos - Similarities and differences. Adv Drug Deliv Rev 2013, 65(15):2078-2086.

5. Dumortier $\mathrm{H}$ : When carbon nanotubes encounter the immune system: desirable and undesirable effects. Adv Drug Deliv Rev 2013, 65(15):2120-2126.

6. Bhattacharya K, Andón FT, El-Sayed R, Fadeel B: Mechanisms of carbon nanotube-induced toxicity: focus on pulmonary inflammation. Adv Drug Deliv Rev 2013, 65(15):2087-2097.

7. Campbell NP, Kindler HL: Update on malignant pleural mesothelioma. Semin Respir Crit Care Med 2011, 32:102-110. Review.

8. Mossman BT, Shukla A, Heintz NH, Verschraegen CF, Thomas A, Hassan R: New insights into understanding the mechanisms, pathogenesis, and management of malignant mesotheliomas. Am J Pathol 2013, 182(4):1065-1077.

9. Vogelzang NJ, Rusthoven JJ, Symanowski J, Denham C, Kaukel E, Ruffie P, Gatzemeier U, Boyer M, Emri S, Manegold C, Niyikiza C, Paoletti P: Phase III study of pemetrexed in cobination with cisplatin versus cisplatin alone in patients with malignant pleural mesothelioma. J Clin Oncol 2003, 21:2636-2644

10. Scherpereel A, Astoul P, Baas P, Berghmans T, Clayson H, de Vuyst $P$, Dienemann H, Galateau-Salle F, Hennequin C, Hillerdal G, Le Péchoux C, Mutti L, Pairon JC, Stahel R, van Houtte P, van Meerbeeck J, Waller D, Weder W: Guidelines of the European Respiratory Society and the European Society of Thoracic Surgeons for the management of malignant pleural mesothelioma. European Respiratory Society/European Society of Thoracic Surgeons Task Force. Eur Respir J 2010, 35(3):479-495.

11. Haas AR, Sterman DH: Malignant pleural mesothelioma: update on treatment options with a focus on novel therapies. Clin Chest Med 2013, 34(1):99-111.

12. Pinton G, Manente AG, Tavian D, Moro L, Mutti L: Therapies currently in Phase II trials for malignant pleural mesothelioma. Expert Opin Investig Drugs 2013, 22(10):1255-1263.

13. Pinton G, Brunelli E, Murer B, Puntoni R, Puntoni M, Fennell DA, Gaudino G, Mutti L, Moro L: Estrogen receptor-beta affects the prognosis of human malignant mesothelioma. Cancer Res 2009, 69:4598-4604.

14. Pillai K, Pourgholami MH, Chua TC, Morris DL: Oestrogen receptors are prognostic factors in malignant peritoneal mesothelioma. $J$ Cancer Res Clin Oncol 2013, 139(6):987-994.

15. Pinton G, Thomas W, Bellini P, Manente AG, Favoni RE, Harvey BJ, Mutti L, Moro $L$ : Estrogen receptor $\beta$ exerts tumor repressive functions in human malignant pleural mesothelioma via EGFR inactivation and affects response to gefitinib. PLOS ONE 2010, 5:e14110.

16. Enmark E, Pelto-Huikko M, Grandien K, Lagercrantz S, Lagercrantz J, Fried G, Nordenskjöld M, Gustafsson JA: Human estrogen receptor beta-gene structure, chromosomal localization, and expression pattern. J Clin Endocrinol Metab 1997, 82:4258-4265.

17. Mersereau JE, Levy N, Staub RE, Baggett S, Zogovic T, Chow S, Ricke WA, Tagliaferri M, Cohen I, Bjeldanes LF, Leitman DC: Liquiritigenin is a plant-derived highly selective estrogen receptor $\beta$ agonist. Mol Cell Endocrinol 2008, 283:49-57.

18. Hartman J, Lindberg K, Morani A, Inzunza J, Ström A, Gustafsson JA: Estrogen receptor $\beta$ inhibits angiogenesis and growth of T47D breast cancer xenografts. Cancer Res 2006, 66:11207-11213.

19. Nilsson S, Koehler KF, Gustafsson JÅ: Development of subtype-selective oestrogen receptor-based therapeutics. Nat Rev Drug Discov 2011, 10:778-792. Review.

20. Thomas C, Gustafsson JA: The different roles of ER subtypes in cancer biology and therapy. Nat Rev Cancer 2011, 11(8):597-608

21. Dey P, Barros RP, Warner M, Strom A, Gustafsson JÅ: Insight into the mechanisms of action of estrogen receptor beta. J Mol Endocrinol 2013, 51(3):T61-T74
22. Murphy $L C$, Leygue $E$ : The role of estrogen receptor- $\beta$ in breast cancer. Semin Reprod Med 2012, 30(1):5-13. Review.

23. Rudolph A, Toth C, Hoffmeister M, Roth W, Herpel E, Jansen L, Marx A, Brenner H, Chang-Claude J: Expression of oestrogen receptor beta and prognosis of colorectal cancer. Br J Cancer 2012, 107:831-839.

24. Gallo D, De Stefano I, Prisco MG, Scambia G, Ferrandina G: Estrogen receptor beta in cancer: an attractive target for therapy. Curr Pharm Des 2012, 18(19):2734-2757.

25. Yakimchuck $\mathrm{K}$, Jondal $\mathrm{M}$, Okret S: Estrogen receptor alpha and beta in the normal immune system and in lymphoid malignancies. Mol Cell Endocrinol 2013, 375:121-129.

26. Yan Y, Li X, Blanchard A, Bramwell VH, Pritchard Kl, Tu D, Shepherd L, Myal Y, Penner C, Watson PH, Leygue E, Murphy LC: Expression of both Estrogen Receptor-beta 1 (ER- $\beta 1$ ) and its co-regulator Steroid Receptor RNA Activator Protein (SRAP) are predictive for benefit from tamoxifen therapy in patients with Estrogen Receptor-alpha (ER-a)-Negative Early Breast Cancer (EBC). Ann Oncol 2013, 24(8):1986-1993.

27. Pillai K, Akhter J, Pourgholami MH, Morris DL: Peritoneal mesothelioma in a woman who has survived for seven years: a case report. J Med Case Rep 2011, 5:36.

28. Barzi A, Lenz A, Labonte MJ, Lenz HJ: Molecular pathways: estrogen pathway in colorectal cancer. Clin Cancer Res 2013, 19(21):5842-5848.

29. Song X, Pan ZZ: Estrogen receptor-beta agonist diarylpropionitrile counteracts the estrogenic activity of estrogen receptor-alpha agonist propylpyrazole-triol in the mammary gland of ovariectomized Sprague Dawley rats. J Steroid Biochem Mol Biol 2012, 130(1-2):26-35.

30. Frasor J, Barnett DH, Danes JM, Hess R, Parlow AF, Katzenellenbogen BS: Response-specific and ligand dose-dependent modulation of estrogen receptor (ER) a activity by ERß in the uterus. Endocrinology 2003, 144:3159-3166.

31. Beral V, Bull D, Green J, Reeves G: Ovarian cancer and hormone replacement therapy in the Million Women Study. Lancet 2007, 369:1703-1710.

32. Ellem SJ, Risbridger GP: Treating prostate cancer: a rationale for targeting local oestrogens. Nat Rev Cancer 2007, 7:621-627. Review.

33. Hillish A, Peters O, Kosemund D, Müller G, Walter A, Schneider B, Reddersen $\mathrm{G}$, Elger W, Fritzemeier KH: Dissecting physiological roles of estrogen receptor $\alpha$ and $\beta$ with potent selective ligands. Mol Endocrinol 2004, 18:1599-1609.

34. Carroll VM, Jeyakumar M, Carlson KE, Katzenellenbogen JA: Diarylpropionitrile (DPN) enantiomers: synthesis and evaluation of estrogen receptor $\beta$-selective ligands. J Med Chem 2012, 55:528-537.

35. Minutolo F, Macchia M, Katzenellenbogen BS, Katzenellenbogen JA: Estrogen receptor $\beta$ ligands: recent advances and biomedical applications. Med Res Rev 2011, 31:364-442.

36. Paterni I, Granchi C, Katzenellenbogen JA, Minutolo F: Estrogen receptor alpha (ERa) and beta (ERß): Subtype-selective ligands and clinical potential. Steroids 2014, Epub ahead of print.

37. Attia DM, Ederveen AG: Opposing roles of ERa and ER $\beta$ in the genesis and progression of adenocarcinoma in the rat ventral prostate. Prostate 2012, 72:1013-1022.

38. Giroux V, Bernatchez G, Carrier JC: Chemopreventive Effect of ERßselective agonist on intestinal tumorigenesis in $\mathrm{Apc}^{\mathrm{Min} /+}$ Mice. $\mathrm{Mol}$ Carcinog 2011, 50:359-369.

39. Mancuso M, Leonardi S, Giardullo P, Pasquali E, Borra F, Stefano ID, Prisco MG, Tanori M, Scambia G, Majo VD, Pazzaglia S, Saran A, Gallo D: The estrogen receptor beta agonist diarylpropionitrile (DPN) inhibits medulloblastoma development via anti-proliferative and pro-apototic pathways. Cancer Lett 2011, 308:197-202.

40. Sareddy GR, Nair BC, Gonugunta VK, Zhang QG, Brenner A, Brann DW Tekmal RR, Vadlamudi RK: Therapeutic significance of estrogen receptor $\beta$ agonists in gliomas. Mol Cancer Ther 2012, 11(5):1174-1182.

41. Yakimchuk K, Iravani M, Hasni MS, Rhönnstad P, Nilsson S, Jondal M, Okret S: Effect of ligand-activated estrogen receptor $\beta$ on lymphoma growth in vitro and in vivo. Leukemia 2011, 25:1103-1110.

42. Marzioni M, Torrice A, Saccomanno S, Rychlicki C, Agostinelli L, Pierantelli I, Rhönnstad P, Trozzi L, Apelqvist T, Gentile R, Candelaresi C, Fava G, Semeraro R, Benedetti A, Gaudio E, Franchitto A, Onori P, De Minicis S, Carpino G, Kallin E, Alvaro D, Nilsson S: An oestrogen receptor $\beta$-selective agonist exerts anti-neoplastic effects in experimental intrahepatic cholangiocarcinoma. Dig Liver Dis 2012, 44:134-142. 
43. Manente AG, Valenti D, Pinton G, Jithesh PV, Daga A, Rossi L, Gray SG, O'Byrne KJ, Fennell DA, Vacca RA, Nilsson S, Mutti L, Moro L: Estrogen receptor $\beta$ activation impairs mitochondrial oxidative metabolism and affects malignant mesothelioma cell growth in vitro and in vivo. Oncogenesis 2013, 2:e72.

44. Pinton G, Manente AG, Angeli G, Mutti L, Moro L: Perifosine as a potential novel anti-cancer agent inhibits EGFR/MET-AKT axis in malignant pleural mesothelioma. PLOS ONE 2012, 7:e36856.

45. Remon J, Lianes P, Martínez S, Velasco M, Querol R, Zanui M: Malignant mesothelioma: new insights into a rare disease. Cancer Treat Rev 2013, 39:584-591.

46. Calabrese EJ: Cancer biology and hormesis; human tumor cell lines commonly display hormetic (biphasic) dose response. Crit Rev Toxicol 2005, 35(6):463-582.

47. Calabrese EJ: Biphasic dose responses in biology, toxicology and medicine: accounting for their generalizability and quantitative features. Environ Pollut 2013, 182:452-460.

48. Reid G, Denger S, Kos M, Gannon F: Human estrogen receptor-alpha: regulation by synthesis, modification and degradation. Cell Mol Life SC 2002, 59(5):821-831.

49. Reid G, Hübner MR, Métivier R, Brand H, Denger S, Manu D, Beaudouin J, Ellenberg J, Gannon F: Cyclic, proteasome-mediated turnover of unliganded and liganded ERalpha on responsive promoters is an integral feature of estrogen signaling. Mol Cell 2003, 11(3):695-707.

50. Altomare DA, You H, Xiao GH, Ramos-Nino ME, Skele KL, De Rienzo A, Jhanwar SC, Mossman BT, Kane AB, Testa JR: Human and mouse mesotheliomas exhibit elevated AKT/PKB activity, which can be targeted pharmacologically to inhibit tumor cell growth. Oncogene 2005, 24:6080-6089

51. Gordon GJ, Mani M, Maulik G, Mukhopadhyay L, Yeap BY, Kindler HL, Salgia R, Sugarbaker DJ, Bueno R: Preclinical studies of the proteasome inhibitor bortezomib in malignant pleural mesothelioma. Cancer Chemother Pharmacol 2008, 61:549-558.

52. Arafa e-SA, Zhu Q, Barakat BM, Wani G, Zhao Q, El-Mahdy MA, Wani AA: Tangeretin sensitizes cisplatin-resistant human ovarian cancer cells through downregulation of phosphoinositide 3-kinase/Akt signaling pathway. Cancer Res 2009, 69:8910-8917.

53. Simoncini T, Hafezi-Moghadam A, Brazil DP, Ley K, Chin WW, Liao JK: Interaction of oestrogen receptor with the regulatory subunit of phosphatidylinositol-3-OH kinase. Nature 2000, 407:538-541.

54. Mannella P, Brinton RD: Estrogen receptor protein interaction with phosphatidylinositol 3- kinase leads to activation of phosphorylated Akt and extracellular signal-regulated kinase $1 / 2$ in the same population of cortical neurons: a unified mechanism of estrogen action. J Neurosci 2006, 26(37):9439-9447.

55. Cacciotti P, Libener R, Betta P, Martini F, Porta C, Procopio A, Strizzi L, Penengo L, Tognon M, Mutti L, Gaudino G: SV40 replication in human mesothelial cells induces HGF/Met receptor activation: a model for viral-related carcinogenesis of human malignant mesothelioma. Proc Natl Acad Sci U S A 2001, 98:12032-12037.

56. Pass HI, Stevens EJ, Oie H, Tsokos MG, Abati AD, Fetsch PA, Mew DJ, Pogrebniak HW, Matthews WJ: Characteristics of nine newly derived mesothelioma cell lines. Ann Thorac Surg 1995, 59(4):835-844.

57. R Core Team: R: A Language and Environment for Statistical Computing. Vienna, Austria: R Foundation for Statistical Computing; 2012. ISBN 3-900051-07-0, URL http://www.R-project.org/.

58. Barkhem T, Carlsson B, Nilsson Y, Enmark E, Gustafsson J, Nilsson S: Differential response of estrogen receptor alpha and estrogen receptor beta to partial estrogen agonists/antagonists. Mol Pharmacol 1998, 54(1):105-112.

doi:10.1186/1476-4598-13-227

Cite this article as: Pinton et al: Agonist activation of estrogen receptor beta $(E R \beta)$ sensitizes malignant pleural mesothelioma cells to cisplatin cytotoxicity. Molecular Cancer 2014 13:227.

\section{Submit your next manuscript to BioMed Central and take full advantage of:}

- Convenient online submission

- Thorough peer review

- No space constraints or color figure charges

- Immediate publication on acceptance

- Inclusion in PubMed, CAS, Scopus and Google Scholar

- Research which is freely available for redistribution

Submit your manuscript at www.biomedcentral.com/submit
( Biomed Central 